\title{
Approximate boundary conditions for diffraction by thin transmissive media.*
}

\author{
A D Rawlins ${ }^{\dagger}$
}

December 19, 2005

\footnotetext{
*keywords:Boundary conditions, waves, diffraction theory

${ }^{\dagger}$ Department of Mathematical Sciences , Brunel University, Uxbridge, Midddlesex, UBH 3PH, England,email:anthony.rawlins@brunel.ac.uk
} 


\begin{abstract}
The object of this note is to describe a method that can be used to obtain useful boundary conditions to model various situations that arise in diffraction theory. In particular when wanting to apply the Wiener-Hopf technique to diffraction problems that involve thin transmissive media. Transmissive here means that the thin layer medium suffers a change in the physical quantities of density, acoustic velocity, and wave number from the surrounding medium. The present approach can be used to obtain approximate boundary conditions for other physical applications where thin strata of transmissive material arise.
\end{abstract}




\section{Introduction}

Situations often arise where one has to deal with problems that involve the propagation of sound waves through liquid, gaseous, or porous layers. The discontinuity in the density and speed of propagation in these layers can considerably complicate the mathematical analysis of the sound field outside the layer. It is often the case in practice that the external far field effects of the layer is required but not the precise nature of the internal field within the thin layer. With this in mind given that one wants to analyze physical problems of the scattering of sound from a transmissive layer that suffers a change in the physical quantities of density, acoustic velocity, and wave number from the surrounding medium; the first thing one needs to do is to mathematically model it. This requires boundary conditions on the contiguous plane interfaces between the different mediums to be used to find the field everywhere in space. However if the layer is in some sense thin, and the field inside this thin layer is not required, then we shall show that the thin layer can be replaced by a discontinuous boundary condition on a line which lies along the centre line of the thin layer. In this work we present the method that can produce the differential boundary conditions which can be used to replace thin transmissive media. This considerably simplifies subsequent mathematical analysis of the physical problem, because it avoids having to deal with the region inside the thin medium. The present approach can be used in other branches of the physical sciences, for example, hydrodynamics, elasticity and electromagnetism. Here we shall describe this method by considering two problems from acoustics. The first problem is where a sound wave travels through and is reflected back from a thin layer. In the second problem the sound wave is reflected back off a thin layer which lies above an impenetrable surface. The methods derives the boundary conditions by considering the transmission of a plane wave through the thin layer. This boundary condition will then hold for any type of wave that consists of a linear superposition of plane waves incident on a thin layer.

\section{A thin transparent layer between two other media}

Consider the situation where a plane pressure acoustic wave $e^{-i k\left(x \cos \theta_{0}+(z-d / 2) \sin \theta_{0}\right)}$ , with time harmonic variation $e^{-i \omega t}$, is incident upon a thin trasmissive medium. The angle of incidence $\theta_{0}$ is the angle between the wave propagation direction and the upper surface of the layer. The transmissive layer is infinite in the $y$-direction, and the origin of the horizontal $(x)$ and $\operatorname{vertical}(z)$ axes is located at the centre of the layer of thickness $d$. The physical quantities inside the thin layer will have subscript 1 , whereas those outside the layer will have no subscript. WE shall denote the density of the region outside (inside) the layer by $\rho\left(\rho_{1}\right)$ and the sound speed there by $c\left(c_{1}\right)$; the corresponding wave number is given by $k=\omega / c\left(k_{1}=\omega / c_{1}\right)$. The transmissive region is thin in comparison to the wavelength of the incident wave, $k d<<1$. The geometry of the situation is 
shown in Fig. 1.

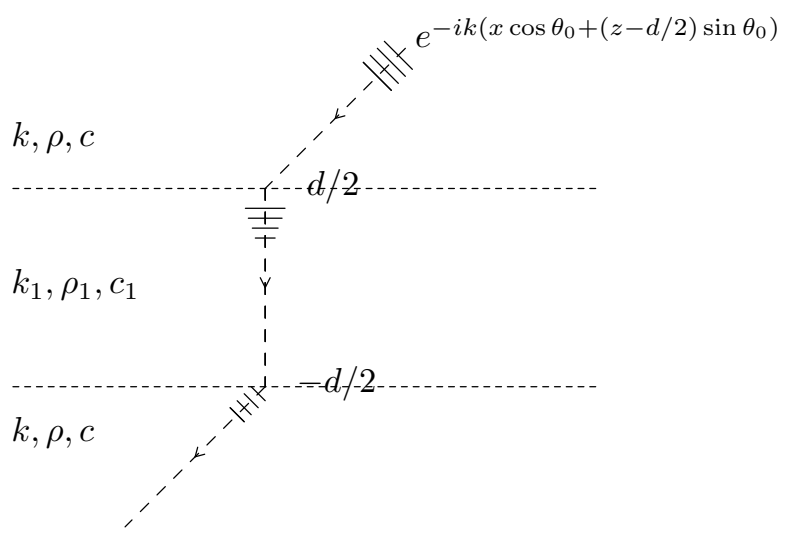

Figure 1:

The pressure field inside the thin layer can be written as $p_{1}(x, z)=A e^{-i k_{1}\left(x \cos \theta_{1}-z \sin \theta_{1}\right)}+B e^{-i k_{1}\left(x \cos \theta_{1}+z \sin \theta_{1}\right)}, \quad-d / 2<z<d / 2$

(1)

where from Snell's law we have $k \cos \theta_{0}=k_{1} \cos \theta_{1}$.

Thus denoting by $p(x, \pm d / 2) \equiv p\left(0^{ \pm}\right)$we have

$$
\begin{gathered}
p_{1}\left(0^{+}\right)=e^{-i k_{1} x \cos \theta_{1}}\left[A e^{i k_{1} \frac{d}{2} \sin \theta_{1}}+B e^{-i k_{1} \frac{d}{2} \sin \theta_{1}}\right], \\
\frac{\partial p_{1}\left(0^{+}\right)}{\partial z}=i k_{1} \sin \theta_{1} e^{-i k_{1} x \cos \theta_{1}}\left[A e^{i k_{1} \frac{d}{2} \sin \theta_{1}}-B e^{-i k_{1} \frac{d}{2} \sin \theta_{1}}\right] \\
p_{1}\left(0^{-}\right)=e^{-i k_{1} x \cos \theta_{1}}\left[A e^{-i k_{1} \frac{d}{2} \sin \theta_{1}}+B e^{i k_{1} \frac{d}{2} \sin \theta_{1}}\right] \\
\frac{\partial p_{1}\left(0^{-}\right)}{\partial z}=i k_{1} \sin \theta_{1} e^{-i k_{1} x \cos \theta_{1}}\left[A e^{-i k_{1} \frac{d}{2} \sin \theta_{1}}-B e^{i k_{1} \frac{d}{2} \sin \theta_{1}}\right]
\end{gathered}
$$

We now require that the pressure wave field outside the thin layer matches the pressure field inside the layer which results in the satisfaction of the boundary conditions: 


$$
\begin{array}{ll}
p\left(0^{+}\right)=p_{1}\left(0^{+}\right), & \frac{1}{\rho_{1}} \frac{\partial p_{1}\left(0^{+}\right)}{\partial z}=\frac{1}{\rho_{0}} \frac{\partial p\left(0^{+}\right)}{\partial z} ; \\
p\left(0^{-}\right)=p_{1}\left(0^{-}\right), & \frac{1}{\rho_{1}} \frac{\partial p_{1}\left(0^{-}\right)}{\partial z}=\frac{1}{\rho_{0}} \frac{\partial p\left(0^{-}\right)}{\partial z} .
\end{array}
$$

These boundary conditions enable us to express the internal pressure field within the layer in terms of the external pressure field outside the layer. Thus from (2) and (3) with (6) we get the system of equations

$$
e^{i k_{1} x \cos \theta_{1}}\left[\begin{array}{c}
p\left(0^{+}\right) \\
\frac{\partial p\left(0^{+}\right)}{\partial z}
\end{array}\right]=\left[\begin{array}{cc}
e^{i k_{1} \frac{d}{2} \sin \theta_{1}} & e^{-i k_{1} \frac{d}{2} \sin \theta_{1}} \\
\left(\frac{\rho_{0}}{\rho_{1}}\right) i k_{1} \sin \theta_{1} e^{i k_{1} \frac{d}{2} \sin \theta_{1}} & -\left(\frac{\rho_{0}}{\rho_{1}}\right) i k_{1} \sin \theta_{1} e^{-i k_{1} \frac{d}{2} \sin \theta_{1}}
\end{array}\right]\left[\begin{array}{c}
A \\
B
\end{array}\right] .
$$

Similarly from (4) (5), and (7) we have

$$
e^{i k_{1} x \cos \theta_{1}}\left[\begin{array}{c}
p\left(0^{-}\right) \\
\frac{\partial p\left(0^{-}\right)}{\partial z}
\end{array}\right]=\left[\begin{array}{cc}
e^{-i k_{1} \frac{d}{2} \sin \theta_{1}} & e^{i k_{1} \frac{d}{2} \sin \theta_{1}} \\
\left(\frac{\rho_{0}}{\rho_{1}}\right) i k_{1} \sin \theta_{1} e^{-i k_{1} \frac{d}{2} \sin \theta_{1}} & -\left(\frac{\rho_{0}}{\rho_{1}}\right) i k_{1} \sin \theta_{1} e^{i k_{1} \frac{d}{2} \sin \theta_{1}}
\end{array}\right]\left[\begin{array}{c}
A \\
B
\end{array}\right] .
$$

Eliminating the matrix involving A and B from (8) and (9) gives

$$
\left[\begin{array}{c}
p\left(0^{+}\right) \\
\frac{\partial p\left(0^{+}\right)}{\partial z}
\end{array}\right]=\left[\begin{array}{cc}
\cos \left(k_{1} d \sin \theta_{1}\right) & \left(\frac{\rho_{1}}{\rho_{0}}\right) \frac{\sin \left(k_{1} d \sin \theta_{1}\right)}{k_{1} \sin \theta_{1}} \\
-\left(\frac{\rho_{0}}{\rho_{1}}\right) k_{1} \sin \theta_{1} \sin \left(k_{1} d \sin \theta_{1}\right) & \cos \left(k_{1} d \sin \theta_{1}\right)
\end{array}\right]\left[\begin{array}{c}
p\left(0^{-}\right) \\
\frac{\partial p\left(0^{-}\right)}{\partial z}
\end{array}\right] .
$$

Thus we have now related the pressure field external to the thin layer on either side of the layer. This means we can solve problems in the region outside the layer without regard to what is happening to the wave field inside the layer. We notice that the $2 \times 2$ matrix in the last equation is similar to the transmission line matrix that occurs in electrical engineering. It will also be observed that the matrix elements depend on $\theta_{1}$ and therefore, from Snell's law, on the angle of the incident plane wave. If the thin layer has some absorption the dependence on the incident angle becomes weakened. Since $k_{1}=k n$ where $n$ is the acoustic refractive index of the thin layer, then we can write

$$
\sin \theta_{1}=\sqrt{1-\left(\frac{\cos \theta_{0}}{n}\right)^{2}} .
$$

If the layer has absorption then $\Im n>0$, and for large absorption $\Im n>0$ and $|n| \gg 1$ in which case

$$
k_{1} \sin \theta_{1}=k n \sqrt{1-\left(\frac{\cos \theta_{0}}{n}\right)^{2}}=k n+O\left(\frac{1}{n}\right),
$$

and the result (10)becomes 


$$
\left[\begin{array}{c}
p\left(0^{+}\right) \\
\frac{\partial p\left(0^{+}\right)}{\partial z}
\end{array}\right]=\left[\begin{array}{cc}
\cos (k n d) & \left(\frac{\rho_{1}}{\rho_{0}}\right) \frac{\sin (k n d)}{k n} \\
-\left(\frac{\rho_{0}}{\rho_{1}}\right) k n \sin (k n d) & \cos (k n d)
\end{array}\right]\left[\begin{array}{c}
p\left(0^{-}\right) \\
\frac{\partial p\left(0^{-}\right)}{\partial z}
\end{array}\right] .
$$

We notice that now the boundary condition is independent of $\theta_{0}$. This is because if $\Im n>0$ and $|n| \gg 1$ irrespective of the angle of incidence, the wave fronts in the layer all travel normal to the surface of the thin layer, see Fig. 2. Geometrically the boundary condition (11), shows that in one dimentional wave propagation with wavenumber $k n$, the vector $\left[p,(k n)^{-1} \partial p / \partial z\right]$ rotates through an angle $k n$ per unit distance.

This type of boundary condition was used successfully in conjunction with the Wiener-Hopf technique to solve the problems of the diffraction of a plane wave by a semi-infinite thin transparent dielectric [1]; and for an acoustically porous barrier with a finite slit [2] The derivation of the boundary condition was achieved in [1] by using a symmetry and reciprocity argument; which is not as straightforward as the present approach. The present approach can also be used to deal with a thin layer that consists of thinner multiple layered strata, each strata consisting of different media. The final boundary condition will be of the form (11), where the elements of the matrix will be a function of the various physical properties of the sub strata. This type of situation can model a thin transparent layer whose physical properties vary continuously across the thin layer.

\section{A thin transparent layer on top of a rigid plane.}

Consider the situation where a plane acoustic wave is incident upon a thin transmissive medium that lies on top of a rigid plane. The layer is infinite in the $y$-direction, and the origin of the $\operatorname{horizontal}(x)$ and $\operatorname{vertical}(z)$ axes is located at the bottom of the layer of thickness $h$. The transparent region is thin in comparison to the wavelength of the incident wave, $k h<<1$. The geometry of the situation is shown in Fig. 2.

The field inside the thin layer of thickness $h$ can be written as

$$
p_{1}(x, z)=A e^{-i k_{1}\left(x \cos \theta_{1}-z \sin \theta_{1}\right)}+B e^{-i k_{1}\left(x \cos \theta_{1}+z \sin \theta_{1}\right)}, \quad 0<z<h ;
$$

whereas the field above the layer can be written as

$$
p(x, z)=e^{-i k\left(x \cos \theta_{0}+(z-h) \sin \theta_{0}\right)}+R e^{-i k\left(x \cos \theta_{0}-(z-h) \sin \theta_{0}\right)}, \quad z>h ;
$$

where from Snell's law we have $k \cos \theta_{0}=k_{1} \cos \theta_{1}$. The angle of incidence $\theta_{0}$ is the angle between the incident wave propagation direction and the upper surface of the layer. 


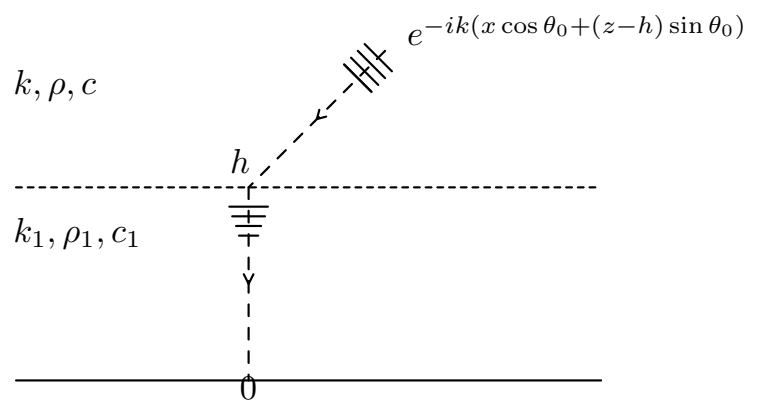

Figure 2:

Thus denoting by $p(x, h) \equiv p\left(0^{+}\right)$we have

$$
p_{1}\left(0^{+}\right)=e^{-i k_{1} x \cos \theta_{1}}\left[A e^{i k_{1} h \sin \theta_{1}}+B e^{-i k_{1} h \sin \theta_{1}}\right],
$$

and

$$
\frac{\partial p_{1}\left(0^{+}\right)}{\partial z}=i k_{1} \sin \theta_{1} e^{-i k_{1} x \cos \theta_{1}}\left[A e^{i k_{1} h \sin \theta_{1}}-B e^{-i k_{1} h \sin \theta_{1}}\right] .
$$

On the surface of the rigid plane we must have $\frac{\partial p_{1}(0)}{\partial z}=0$ so that from (15) we get

$$
\frac{\partial p_{1}(0)}{\partial z}=i k_{1} \sin \theta_{1} e^{-i k_{1} x \cos \theta_{1}}[A-B]=0 .
$$

From (16)we have $A=B$ so that (14) and (15) become

$$
\begin{gathered}
p_{1}\left(0^{+}\right)=e^{-i k_{1} x \cos \theta_{1}} 2 A \cos \left(k_{1} h \sin \theta_{1}\right) ; \\
\frac{\partial p_{1}\left(0^{+}\right)}{\partial z}=-k_{1} \sin \theta_{1} e^{-i k_{1} x \cos \theta_{1}} 2 A \sin \left(k_{1} h \sin \theta_{1}\right) .
\end{gathered}
$$

Hence eliminating $A$ from the last two equations gives the impedance type boundary condition:

$$
\frac{\partial p_{1}\left(0^{+}\right)}{\partial z}+k_{1} \sin \theta_{1} \tan \left(k_{1} h \sin \theta_{1}\right) p_{1}\left(0^{+}\right)=0 .
$$

which becomes, on using the boundary condition on the upper surface of the thin layer, ie (6) 


$$
\frac{\partial p\left(0^{+}\right)}{\partial z}+\frac{k_{1} \rho_{0}}{\rho_{1}} \sin \theta_{1} \tan \left(k_{1} h \sin \theta_{1}\right) p\left(0^{+}\right)=0 .
$$

Thus the thin transparent layer is replaced by an impedance type boundary condition. If the layer has absorption ( $\Im n>0$ and $|n| \gg 1$ )this boundary condition becomes

$$
\frac{\partial p\left(0^{+}\right)}{\partial z}+\frac{k n \rho_{0}}{\rho_{1}} \tan (k n h) p\left(0^{+}\right)=0 .
$$

\section{Conclusions}

We have presented a simple systematic method which enables one to derive approximate boundary conditions outside thin transmissive strata that avoids having to consider the region inside the strata. This considerably simplifies the mathematical analysis of problems that involve wave scattering by such surfaces, especially if they are of semi-infinite or finite length. The method can be applied to thin layers that consist of thinner sub-strata, and therefore can deal with thin layers whose material properties vary across the thin layer in a smooth manner. A further technical point that is worth making is that this approach yields boundary conditions that involve derivatives of a lower order than those of the wave equation and therefore avoids uniqueness problems that that approximate boundary conditions of a higher order would involve.

\section{References}

[1] A D Rawlins. Diffraction by an acoustically penetrable or an electromagnetically dielectric half plane. Int. J.Engng. Sci. (15), 1977, 569-578.

[2] S Asghar,T Hayat,and J G Harris. Diffraction by a slit in an infinite porous barrier. Wave Motion. (33), 2001, 24-40. 
Legend to fig1: A plane wave incident on a thin transmissive layer.

Legend to fig2: Plane wave incident on a thin transmissive layer above a rigid plane. 